\title{
Les structures interrogatives directes partielles fondées sur où dans les dialogues de romans français du XXe siècle
}

\author{
Nathalie Rossi-Gensane*, Luisa Fernanda Acosta Córdoba ${ }^{\star}$, Biagio Ursi ${ }^{* *}$ et Margot Lambert \\ *Université Lumière Lyon 2 \& ICAR (UMR 5191) and ${ }^{* * A i x-M a r s e i l l e ~ U n i v e r s i t e ́ ~ \& ~ L P L ~(U M R ~ 7309) ~}$ \\ Email: Nathalie.Rossi-Gensane@univ-lyon2.fr
}

(Received 11 February 2020; revised 30 August 2020; accepted 24 September 2020; first published online 25 January 2021)

\begin{abstract}
Résumé
Nous appuyant sur un corpus de 2731 occurrences constitué au sein de Frantext à partir de 1250 textes (soit 78063933 mots), nous proposons un panorama de diverses structures interrogatives directes partielles fondées sur où dans les dialogues de romans français entre 1900 et 1997. Nous relions ces structures à des facteurs tels que la date de l'œuvre, le sexe, l'âge, l'appartenance sociale et l'origine géographique du personnage auquel est attribué le discours direct, etc., en envisageant également la situation de communication fictionnelle. L'article entend, à partir d'études existantes sur l'oral réel, apprécier l'écart - et son évolution - entre celui-ci et l'oral mis en scène à l'écrit, avec un éclairage quantitatif inédit. Malgré l'absence d'études sur l'oral réel dans la première moitié du XX $\mathrm{XX}^{\mathrm{e}}$ siècle, il peut être souligné en fin de siècle un réalisme accru, les normes objectives de l'oral mis en scène allant dans le sens (généralement avec décalage) de celles de l'oral réel. Peut alors aussi être mise en évidence une plus grande prise en compte de la dimension diaphasique au détriment de la dimension diastratique.
\end{abstract}

\section{Introduction ${ }^{1}$}

Comme le signale, parmi de nombreux auteurs, Coveney (2011: 112), "L'interrogation directe est un domaine qui connaît une très grande diversité de structures, ainsi qu'une distribution complexe de ces structures, conditionnée par des facteurs linguistiques, pragmatiques et sociostylistiques ». Nous appuyant sur un corpus de 2731 occurrences constitué au sein de Frantext ${ }^{2}$ à partir de 1250 textes (soit 78063933 mots), nous proposons un panorama de diverses structures interrogatives directes partielles fondées sur l'adverbe où, posant une

\footnotetext{
${ }^{1}$ Nous remercions vivement Elissa Pustka, David Hornsby et Andreas Dufter, ainsi que les relecteurs anonymes, pour leurs remarques.

${ }^{2}$ http://www.frantext.fr/

(c) The Author(s), 2021. Published by Cambridge University Press. This is an Open Access article, distributed under the terms of the Creative Commons Attribution licence (http://creativecommons.org/licenses/by/4.0/), which permits unrestricted re-use, distribution, and reproduction in any medium, provided the original work is properly cited.
} 
question sur le lieu ${ }^{3}$, dans les dialogues de romans français entre 1900 et 1997. Il s'agit donc d' « oral représenté », selon l'expression de Marchello-Nizia (2012), ou encore d'oral mis en scène à l'écrit, recouvrant " des séquences écrites qui se donnent de manière explicite comme la restitution de paroles orales » et qui fournissent l' " accès à une oralité construite à l'intérieur de l'écrit " (Guillot-Barbance et al. 2017: 54). Nous entendons comparer cet oral mis en scène à l'écrit avec l'oral réel, tel qu'il a été caractérisé à partir des années 1960 dans certaines études empiriques (Behnstedt 1973, Söll 1982, Coveney 1996/2002, Druetta 2009).

Dans le cadre de cet article, nous nous sommes cantonnés aux structures suivantes, présentes dans une proportion d'au moins $10 \%$ dans l'un des quatre quarts de siècle (Tableau 1):

Tableau 1. Les structures interrogatives étudiées ${ }^{4}$

\begin{tabular}{ll}
\hline Type & Exemple \\
\hline (Prép) où V CL & Où va-t-il ? / Par où passe-t-il ? \\
\hline (Prép) où S V & Où il/ton frère va ? / Par où il/ton frère passe ? \\
\hline S V (prép) où & II/Ton frère va où ? / II/Ton frère passe par où ? \\
\hline (Prép) où que ${ }^{5}$ & Où qu'/e il/ton frère va ? / Par où qu'/e il/ton frère passe ? \\
\hline
\end{tabular}

Nous avons toutefois exclu les structures (prép) où $\mathrm{V} \mathrm{N}$ (où va ton frère? / par où passe ton frère ?) et (prép) où sans $\mathrm{V}$ (où ? / par où ?), qui répondaient à cette condition mais n'offraient pas une base de comparaison suffisante avec l'oral réel (la première y étant peu représentée et la seconde étant peu documentée dans les enquêtes). N’ont pas été non plus traitées les structures « rares » telles que l'inversion complexe (où ton frère va-t-il ? / par où ton frère passe-t-il ?), ou celles centrées sur un verbe non conjugué (où aller? / par où passer ?), ou encore celles en est-ce que (où est-ce qu'/e il/ton frère va? / par où est-ce qu'/e il/ton frère passe ?). ${ }^{6}$

\footnotetext{
${ }^{3}$ Nous avons fait le choix d'examiner de manière approfondie, en diachronie, un seul interrogatif. Où présente de plus l'intérêt d'être doté d'une certaine polyvalence syntaxique, pouvant notamment être complément simplement régi (Où marche-t-il ?) et complément de verbe (Où va-t-il ?) (voir 4.2 infra).

${ }^{4}$ Les conventions adoptées (dans tout l'article) sont les suivantes: prép $=$ préposition, $\mathrm{V}=$ verbe, $\mathrm{CL}=$ pronom clitique, $\mathrm{N}=$ nom ou pronom non clitique, $\mathrm{S}=$ sujet. Précisons que, quand un déterminant accompagne un nom, celui-là n'est pas indiqué par souci de simplicité. Les parenthèses encadrent un élément facultatif.

${ }^{5} \mathrm{Il}$ s'agit ici de plusieurs structures débutant par (prép) où que, qui comptent aussi, au-delà de (prép) où que $\mathrm{S} \mathrm{V}$, dont des exemples sont donnés, où que $\mathrm{V} \mathrm{N}$, où qu'il y $a \mathrm{~N}$, où que sans $\mathrm{V}$ et où que c'est que $\mathrm{CL} \mathrm{V}$ (voir Section 6 infra).

${ }^{6} \mathrm{Au}$ premier abord, la rareté des structures en est-ce que (autour de $1 \%$ de l'ensemble des interrogatives directes en où durant tout le $\mathrm{XX}^{\mathrm{e}}$ siècle) peut paraître surprenante car ces structures sont signalées comme répandues dans certaines enquêtes sur l'oral réel, telle celle de Coveney (1996/2002) où, tous interrogatifs confondus (désignés par Q), Q est-ce que S V atteint 48,4\% (voir 2.1 infra). Cependant, d'une part, Coveney (2002: 231) précise que, dans 45 cas sur 105, l'interrogatif est que; autrement dit, il s'agit alors de qu'est-ce que. D'autre part, Guryev (2017: 235 et 226) indique, pour son corpus suisse de SMS, un taux assez bas (5,6\%) de la structure Q est-ce que S V dont, selon lui, « l'emploi [...] diminue quand on passe du médium phonique au médium graphique». Mentionnons aussi que la structure $\mathrm{Q}$ est-ce que $\mathrm{S} \mathrm{V}$ a la fréquence la plus élevée pour les interrogatives partielles dans le corpus de dialogues de films de Dekhissi (2013), mais, là encore, en raison du grand nombre d'occurrences de qu'est-ce que (voir Dekhissi 2013: 113).
} 
À la suite, notamment, de Houdebine (2015: 14 et 15), nous nous appuierons sur la distinction entre normes objectives " dégagées par la description ", adossées à des fréquences ou des tendances, et normes subjectives associées à des jugements de valeur, dont font partie les normes prescriptives " étayées par un discours antérieur de type institutionnel, scolaire ou grammatical ». Nous apprécierons la congruence, ou pas, entre les normes objectives de l'oral réel et celles de l'oral mis en scène à l'écrit, ainsi que la place des normes subjectives, généralement davantage à l'œuvre pour l'écrit littéraire.

Il est parfois avancé que, tandis que la variation phonologique est liée à des facteurs sociodémographiques (sexe, âge, appartenance sociale, origine géographique, etc.), pour la syntaxe seraient "surtout [en jeu] des facteurs linguistiques ou situationnels, guère [...] des facteurs sociaux, dans la mesure où [la] moindre fréquence [des phénomènes] les rend moins saillants pour un investissement social » (Gadet 1997 : 17). En nous concentrant sur le début et la fin du XX siècle, nous nous interrogerons sur les parts respectives de la dimension diastratique et de la dimension diaphasique pour ces structures interrogatives relativement répandues dans l'oral mis en scène. ${ }^{7}$ Nous nous demanderons notamment s'il n'y aurait pas, en fin de siècle, évolution en faveur du diaphasique. En effet, la quasi-disparition des structures en que, vouées diastratiquement au stéréotypage du parler populaire, s'accompagne alors de l'essor de deux structures, (prép) où S V et S V (prép) où, avant tout marquées diaphasiquement comme familières.

Dans ce qui suit, après avoir rappelé les tendances mises au jour par des études effectuées depuis les années 1960 sur l'oral réel, précisé l'évaluation sociostylistique des différentes structures interrogatives et présenté notre corpus d'oral mis en scène et nos choix méthodologiques (Section 2), nous apprécierons sur le plan linguistique, puis, plus brièvement, sur le plan des caractéristiques des personnages et des situations de communication fictionnelles, l'écart (et son évolution) entre oral réel et oral mis en scène à l'écrit pour quatre sortes de structures interrogatives. Nous examinerons ainsi tout d'abord la structure la plus répandue dans l'oral représenté, la postposition clitique (prép) où V CL (Section 3), puis les structures en augmentation dans l'oral représenté, (prép) où S V (Section 4) et S V (prép) où (Section 5), enfin, les structures en diminution dans l'oral représenté en que (Section 6). Nous entendons montrer qu'en fin de siècle, l'oral mis en scène reflète plus fidèlement l'oral réel, les normes objectives du premier allant dans le sens (généralement avec décalage) de celles du second et les normes subjectives semblant occuper une place moindre. Sera aussi soulignée une plus grande prise en compte de la dimension diaphasique au détriment de la dimension diastratique.

\section{Corpus et méthodologie}

\section{1. Études empiriques sur l'oral réel}

Avant de nous pencher (à partir de la Section 3) sur l'oral mis en scène à l'écrit, dans les dialogues de romans français du $\mathrm{XX}^{\mathrm{e}}$ siècle, nous évoquerons tout d'abord

\footnotetext{
${ }^{7}$ La dimension diatopique sera peu évoquée (voir toutefois 6.3 infra), notre corpus d'oral mis en scène à l'écrit se prêtant moins bien à une étude systématique sur ce plan.
} 
certaines études antérieures sur l'oral réel ${ }^{8}{ }^{8}$ en écartant néanmoins celles sur le français canadien (cf. par exemple Elsig 2009), dans la mesure où « l'interrogation est un des domaines où les variétés canadiennes et américaines se distinguent le plus du français de référence " (Coveney 2011: 133).

Coveney (2011: 126) résume dans le tableau 2, que nous avons adapté, ${ }^{9}$ les résultats d'enquêtes quantitatives effectuées depuis les années 1960:

Tableau 2. «Fréquence des principales variantes de l'interrogation partielle (deuxième moitié du XX siècle) ", d'après Coveney (2011: 126) $)^{10}$

\begin{tabular}{|c|c|c|c|c|c|c|c|c|}
\hline Variété & Enquête & $\begin{array}{c}\text { Nombre } \\
\text { d'occurrences }\end{array}$ & $\begin{array}{l}\mathrm{S} V \\
\mathrm{Q}^{11}\end{array}$ & Q S V & $\begin{array}{l}\text { Q que } \\
\text { S V }\end{array}$ & $\begin{array}{c}\text { Q est-ce que } \\
\text { S V }\end{array}$ & $\begin{array}{l}\text { Q V } \\
\mathrm{CL}\end{array}$ & $\begin{array}{l}\text { Q V } \\
\text { N }\end{array}$ \\
\hline Radio & $\begin{array}{l}\text { Behnstedt } \\
1973\end{array}$ & 4367 & $25 \%$ & $10 \%$ & $0 \%$ & $3 \%$ & $50 \%$ & $12 \%$ \\
\hline $\begin{array}{l}\text { Bourgeois } \\
\text { familier }\end{array}$ & $\begin{array}{l}\text { Behnstedt } \\
1973\end{array}$ & 446 & $33 \%$ & $46 \%$ & $0 \%$ & $12 \%$ & $3 \%$ & $2 \%$ \\
\hline $\begin{array}{l}\text { Enfants } \\
\text { (9 ans) }\end{array}$ & Söll 1982 & 364 & $12.9 \%$ & $31.6 \%$ & $6.9 \%$ & $41.5 \%$ & & $.6 \%$ \\
\hline Ouvriers & $\begin{array}{l}\text { Behnstedt } \\
1973\end{array}$ & 587 & $12 \%$ & $36 \%$ & $26 \%$ & $8 \%$ & & $9 \%$ \\
\hline
\end{tabular}

Ces enquêtes peuvent être complétées par celles, plus récentes, de Coveney (1996/2002) et de Druetta (2009). L'étude de Coveney (1996/2002) se fonde sur un corpus d'entretiens informels avec des locuteurs originaires de Picardie. Celle de Druetta (2009) est basée sur des corpus également de type conversationnel: des corpus de français parlé recueillis et transcrits par l'équipe du Groupe Aixois de Recherche en Syntaxe d'Aix-en-Provence et un corpus radiophonique recueilli par l'auteur. Les résultats sont présentés dans les tableaux 3 et 4 , respectivement tiré de Coveney (2002: 118) et adapté de Druetta (2009: 158):

Coveney (2002: 108-109) note, pour les études de Behnstedt (1973) et de Söll (1982), que la structure S V Q est utilisée dans plusieurs situations de communication et par tous les groupes (radio (des années 1960-1970), bourgeois familier, enfants, ouvriers). Elle est également bien représentée dans l'enquête de Coveney (1996/2002), ainsi que dans celle de Druetta (2009) où elle apparaît en premier. La structure Q S V, dans les études de Behnstedt (1973) et de Söll

\footnotetext{
${ }^{8}$ On signalera également la fiche FRACOV intitulée « Interrogation » (Lefeuvre et Rossi-Gensane 2015), qui propose une présentation synthétique détaillée des interrogatives directes dans l'oral réel à partir de sept corpus.

${ }^{9}$ Nous n'avons pas mentionné les résultats de l'enquête menée en 1970 par Terry car, s'appuyant sur un corpus de théâtre (de boulevard), elle ne concerne pas l'oral réel.

${ }^{10}$ Pour une présentation détaillée de ces enquêtes, on se reportera à Coveney (2002: 105-106). Par ailleurs, Coveney (2011: 126-127) précise que, dans ce tableau, « les chiffres [ont été] omis pour quelques variantes 'mineures', à savoir $\mathrm{Q}$ que c'est que $\mathrm{S} \mathrm{V}, \mathrm{Q}$ c'est que $\mathrm{S} \mathrm{V}$ et c'est $\mathrm{Q}$ que $\mathrm{S} \mathrm{V}$, ce qui explique le fait que, pour quelques variétés, le total des fréquences relatives ne soit pas $100 \%$ ».

${ }^{11}$ Rappelons que Q (pour mot qu-) désigne l'interrogatif.
} 
Tableau 3. Fréquence des variantes de l'interrogation partielle dans l'enquête de Coveney (1996/2002)

\begin{tabular}{lcccccc}
\hline $\begin{array}{l}\text { Nombre } \\
\text { d'occurrences }\end{array}$ & S V Q & Q S V & $\begin{array}{l}\text { Q est-ce } \\
\text { que S V }\end{array}$ & Q V CL & Q V N & Q=S V \\
\hline 122 & $15,6 \%$ & $23,8 \%$ & $48,4 \%$ & $6,6 \%$ & $2,5 \%$ & $3,3 \%$ \\
\hline
\end{tabular}

Tableau 4. Fréquence des variantes de l'interrogation partielle dans l'enquête de Druetta (2009)

\begin{tabular}{lccccc}
\hline $\begin{array}{l}\text { Nombre } \\
\text { d'occurrences }\end{array}$ & S V Q & Q S V & $\begin{array}{c}\text { Q est-ce que (y compris } \\
\text { qu'est-ce qui et } \\
\text { qu'est-ce que) }\end{array}$ & $\begin{array}{c}\text { Q V S ou } \\
\text { Q = S V }{ }^{12}\end{array}$ & Q sans V \\
\hline 406 & $29 \%$ & $22,66 \%$ & $16 \%$ & $12,8 \%$ & $19,45 \%$ \\
\hline
\end{tabular}

(1982), figure dans le corpus radiophonique, mais surtout « dans les trois corpus de langue spontanée " (Coveney 2011: 127), et particulièrement dans la variété de bourgeois familier de Behnstedt (1973). Cette structure apparaît aussi en bonne place (chaque fois la deuxième) dans les enquêtes de Coveney (1996/2002) et de Druetta (2009). Dans les études de Behnstedt (1973) et de Söll (1982), la structure Q que S V est totalement absente du corpus radiophonique et de la variété de bourgeois familier, et produite dans une faible mesure par les enfants et dans une assez grande mesure par les ouvriers. Elle est également absente dans les enquêtes de Coveney (1996/2002) et de Druetta (2009). La structure Q est-ce que $\mathrm{S} \mathrm{V}$ est présente de manière minoritaire dans toutes les variétés des enquêtes de Behnstedt (1973) et dans l'enquête de Druetta (2009), de manière prépondérante dans les enquêtes de Söll (1982) et de Coveney (1996/2002) (avec presque la moitié des occurrences dans cette dernière). L'inversion clitique, extrêmement présente (à hauteur de 50\%) dans le corpus radiophonique datant des années 1960-1970, est, comme le révèlent tous les autres corpus, faiblement produite dans la langue parlée spontanée. ${ }^{13}$ On note cependant que, dans le corpus des enfants (Söll 1982), dans celui des ouvriers (Behnstedt 1973) et dans l'enquête de Druetta (2009), la postposition pronominale clitique n'est pas dissociée de la postposition nominale. Ce dernier procédé, possible pour l'interrogation partielle, est néanmoins assez peu fréquent, hormis pour le corpus radiophonique de Behnstedt (1973) (où il apparaît à hauteur de 12\%). Enfin, Coveney (2011: 126) souligne, pour les enquêtes de Behnstedt (1973) et de Söll (1982), que " l'inversion complexe (N V CL) est extrêmement rare dans ces corpus, parfois même complètement absente ». Il en est également ainsi pour les enquêtes de Coveney (1996/2002) et de Druetta (2009).

\footnotetext{
${ }^{12}$ Dans la structure $\mathrm{Q}=\mathrm{S} \mathrm{V}$, le mot $q u$ - exerce la fonction sujet (Qui vient ?) ou, tout au moins, fait partie du syntagme sujet (Quel homme vient?). Ce cas de figure ne saurait concerner l'interrogatif où.

${ }^{13}$ Coveney (2011: 126) précise que, « dans le corpus des enfants, toutes les occurrences de V CL sauf une (As-tu regardé Télé-Dimanche ?) [sont] en fait des citations de la bande sonore d'un film ».
} 


\section{2. Évaluation sociostylistique des structures interrogatives}

Pour la comparaison entre oral réel et oral mis en scène, il est en outre intéressant de préciser l'évaluation sociostylistique des différentes structures interrogatives, qui participe de la construction des normes subjectives, tout particulièrement prescriptives. Coveney (2011: 124), à la suite de Coveney (2002: 98), propose une synthèse des positions de divers grammairiens dans le tableau 5:

Tableau 5. "Évaluation sociostylistique des variantes de l'interrogation totale et partielle » (Coveney 2011: 124)

\begin{tabular}{lll}
\hline & Totale & Partielle \\
\hline Écrit formel; parlé soutenu & $\mathrm{V} \mathrm{CL}$ & $\mathrm{Q} \vee \mathrm{CL}$ \\
\hline Neutre & & $\mathrm{Q}=\mathrm{S}$ V, Q V N \\
\hline Neutre (mais parfois « inélégant » à l'écrit) & est-ce que S V & Q est-ce que S V \\
\hline Familier (mais non stigmatisé à l'oral) & $\mathrm{S} \mathrm{V}$ & $\mathrm{S} \vee \mathrm{Q}$ \\
\hline Hypercorrection & est-ce que N V CL & $\mathrm{Q}=\mathrm{S} \vee \mathrm{CL}$ \\
\hline Familier/populaire & & $\mathrm{Q}$ S V, c'est Q que S V \\
\hline Populaire & & $\mathrm{Q}$ que S V, Q c'est que S V \\
\hline Rural/populaire & $\mathrm{S} \mathrm{V-ti}$ & \\
\hline
\end{tabular}

On indiquera toutefois une différence avec la classification de Coveney (2002), où la structure Q V N était signalée comme relevant de l'écrit formel et du parlé soutenu.

\subsection{Présentation du corpus d'oral mis en scène et des choix méthodologiques}

Comme il a été mentionné dans l'introduction, nous nous appuyons sur un corpus de 2731 occurrences constitué au sein de Frantext à partir de 1250 textes (soit 78063933 mots). Il s'agit d'occurrences de l'adverbe où dans des interrogatives directes (partielles), issues de dialogues ${ }^{14}$ de romans français entre 1900 et 1997. Nous avons d'abord recherché les occurrences de où éloignées de sept mots ou moins d'un point d'interrogation, grâce à une requête formulée en janvier 2018 et portant sur la partie catégorisée de Frantext. Par la suite, nous avons éliminé manuellement les occurrences non pertinentes (par exemple, les cas où où s'avérait être un pronom relatif dans une phrase interrogative) et trié selon les structures, également manuellement, les 2731 occurrences pertinentes obtenues, qui proviennent de 402 textes.

Chaque interrogative a été classée dans un fichier Excel et systématiquement accompagnée des informations suivantes: le type (la structure) de l'interrogative,

\footnotetext{
${ }^{14}$ La notion de dialogue est entendue dans un sens large. Par exemple, les questions auto-adressées sont incluses dans le corpus. De manière plus générale, les interrogatives, dans certains cas, peuvent relever de paroles rapportées " intérieures".
} 
l'éventuelle réponse reçue, l'œuvre dont provient l'interrogative, la date de (la première) publication de l'œuvre, le nom de l'auteur, la date de naissance de l'auteur. Des informations supplémentaires, quand elles étaient disponibles, le cas échéant dans le reste du roman, ont été renseignées, d'une part, pour les interrogatives de la structure (prép) où V CL issues d'œuvres datées d'entre 1900 et 1908 et d'entre 1985 et 1997 et, d'autre part, pour les interrogatives des structures (prép) où $\mathrm{S} V$ et $\mathrm{S} V$ (prép) où, ainsi que celles débutant par (prép) où que, issues d'œuvres datées d'entre 1900 et 1925 et d'entre 1976 et 1997: pour l'interrogative, sa fonction communicative; pour le personnage locuteur, son nom, son sexe, son âge, son appartenance sociale, son niveau d'éducation et/ou sa profession, ${ }^{15}$ son origine géographique; pour la situation de communication fictionnelle, son côté privé ou public, familier ou formel. ${ }^{16}$

Précisons enfin que nous avons choisi de découper le $\mathrm{XX}^{\mathrm{e}}$ siècle en quatre périodes quasi égales, 1900-1925, 1926-1950, 1951-1975, 1976-1997. Ce découpage, ni trop fin, ni trop grossier, présente en outre l'avantage de recouvrir des étapes d'intégration de l'" oral dans l'écrit » en littérature, tels le " début du $\mathrm{XX}^{\mathrm{e}}$ siècle " où "l'on peut trouver une distinction très marquée entre langage de la narration et langage des dialogues ", les " années trente " où "l'on peut trouver [...] un langage qui, plutôt qu'à reproduire l'oral, cherche à le transposer " et une " époque [plus] récente " (à partir, approximativement, de 1975) où " l'on peut trouver [...] une indifférenciation entre langage de la narration [...] et langage des dialogues » (Rouayrenc 2010: 251-252).

Conformément à ce qui était annoncé à la fin de l'introduction, nous nous pencherons dans ce qui suit sur l'oral mis en scène à l'écrit à travers quatre sortes de structures interrogatives: tout d'abord, la structure qui y est la plus répandue, la postposition clitique (prép) où V CL (Section 3); puis les structures qui y sont en augmentation, (prép) où $\mathrm{S} V$ (Section 4) et $\mathrm{S} V$ (prép) où (Section 5); enfin, les structures en que, qui y sont en diminution (Section 6).

\section{La structure la plus répandue dans l'oral mis en scène: la postposition clitique (prép) où $\mathrm{V} \mathrm{CL}$}

Faiblement produite dans l'oral spontané, comme il a été rappelé en 2.1, la structure traditionnellement dite à inversion simple à sujet clitique, encore dénommée à postposition clitique, est la plus répandue dans l'oral mis en scène, et ce, quelle que soit la période (Tableau 6). Ainsi, elle atteint la majorité absolue dans la première moitié du $\mathrm{XX}^{\mathrm{e}}$ siècle et la frôle dans le troisième quart. Néanmoins, elle subit une forte baisse dans le dernier quart. Il s'agit, pour cette structure, du cas le plus net de non-congruence entre les normes objectives de l'oral réel et celles de l'oral mis en scène, en relation à la plus grande influence sur l'écrit des normes subjectives, notamment prescriptives (voir 2.1 et 2.2 supra).

\footnotetext{
${ }^{15}$ Le niveau d'éducation a été parfois extrapolé à partir de la profession, une vachère ayant par exemple été présumée peu éduquée, à l'inverse d'une institutrice.

${ }^{16}$ Le côté familier ou formel a été évalué sur la base de divers indices, par exemple, dans le premier cas, la présence du tutoiement (voir Coveney 2016 ainsi que Kerbrat-Orecchioni 1992: 45-55), ou encore de ce que Sauvageot (1964) appelle un " lexique parallèle », tel bagnole utilisé plutôt que voiture.
} 
Tableau 6. L'évolution quantitative dans l'oral mis en scène au XXe siècle de la structure (prép) où V CL

\begin{tabular}{|c|c|c|c|c|}
\hline Période (date de l'œuvre) & $1900-1925$ & $1926-1950$ & $1951-1975$ & 1976-1997 \\
\hline Où V CL & 313 & 466 & 279 & 134 \\
\hline Prép où V CL & 43 & 72 & 37 & 22 \\
\hline Pourcentage $^{17}$ & $\begin{array}{c}356 / 649= \\
54,85 \%\end{array}$ & $\begin{array}{c}538 / 1009= \\
53,32 \%\end{array}$ & $\begin{array}{c}316 / 642= \\
49,22 \%\end{array}$ & $\begin{array}{c}156 / 431= \\
36,19 \%\end{array}$ \\
\hline
\end{tabular}

\section{1. État de l'art}

L'évolution de la structure à postposition clitique dans l'oral représenté semble refléter, certes de manière différée, son recul en français parlé au fil du $\mathrm{XX}^{\mathrm{e}}$ siècle, comme le montre « la comparaison entre le relevé de Foulet, au début [de ce] siècle [...], et [les] études des vingt dernières années » (Druetta 2011). Cette diminution dans l'oral mis en scène peut aussi être attribuée à l'assouplissement des normes prescriptives, dans la mesure où, selon Elsig (2009: 29), "It is only towards the end of the twentieth century that grammars admit that Pronominal inversion is the feature of cultivated, literary, or written styles of French (Grevisse 1993: 584, Riegel et al. 1994: 392) ». ${ }^{18}$

Certains facteurs linguistiques, que notamment Elsig (2009: 18-22) a résumés, sont présentés comme privilégiant la postposition clitique, tels les verbes de haute fréquence, que Dewaele (1999: 174), cité par Elsig (2009: 20), réduit même à cinq: avoir, pouvoir, trouver, faire et vouloir. Les verbes modaux et les auxiliaires (pour lesquels, d'ailleurs, il y a souvent recouvrement avec les verbes de haute fréquence) sont aussi généralement vus comme exerçant un rôle positif. Fromaigeat (1938: 40) notait à cet égard: « dans les temps composés [l'inversion] résiste mieux que dans les autres cas ». Il n'existe pas de consensus, selon les auteurs, quant à l'influence, favorable ou défavorable, des pronoms sujets sur l'inversion. Coveney (2002: 190) souligne cependant que la combinaison entre un verbe du premier groupe au présent et un pronom de première personne, du type Continué-je ?, est de plus en plus inacceptable depuis le français classique. Coveney (2002: 190) indique, à partir de quatre grammaires consultées (Grevisse 1986, Mauger 1968, Byrne et Churchill 1986, Price 1971), la liste des verbes encore susceptibles d'inversion avec je, soit, par ordre décroissant d'acceptabilité: suis, ai, puis, dis, dois, sais, vais, fais, vois et veux. Quoi qu'il en soit, comme l'exprimait déjà Foulet (1921: 345), "l'inversion est un procédé qui trouve de moins en moins de faveur ».

\footnotetext{
${ }^{17}$ Le pourcentage résulte du nombre d'interrogatives du type concerné divisé par le nombre total d'interrogatives pour la période.

${ }^{18}$ Par exemple, comme le souligne Elsig (2009: 29), Riegel et al. (1994: 392) précisent que « l'inversion du sujet est surtout réservée à l'écrit, en particulier littéraire ".
} 


\subsection{Facteurs linguistiques}

On commentera plus particulièrement, pour cette structure, les 102 occurrences produites entre 1900 et 1908 et les 65 occurrences produites entre 1985 et 1997 dans notre corpus. Dans le prolongement d'Elsig (2009: 19 et 262), qui souligne que, si certaines structures apparaissent favorisées par des verbes de haute fréquence, c'est une indication du début de la baisse de leur productivité, on remarquera que la plupart des verbes impliqués dans la structure à postposition clitique sont fréquents. En relation spécifique à la proforme où, vient en premier pour les deux périodes le verbe être exprimant une localisation statique (et équivalant alors à se trouver), suivi par le verbe de localisation dynamique aller (toutefois, dans ce cas, dans une moindre mesure pour la dernière période). On remarquera aussi la plus forte présence de verbes modaux pour la dernière période (où ils participent d'ailleurs trois fois d'une séquence plus ou moins figée où veux-tu/voulez-vous en venir, contre une fois pour la première période).

Pour ce qui est des temps et des modes, on notera, pour la dernière période, l'augmentation tant absolue que relative des temps composés ou périphrastiques (12 sur 65, vs 9 sur 102 pour la première période), et donc un plus fort recours aux verbes auxiliaires. Le temps le plus représenté reste néanmoins le présent, avec à peu près $3 / 4$ des occurrences pour la première période et $2 / 3$ pour la dernière période.

Pour ce qui est des pronoms sujets, leur ordre diffère dans les deux périodes (Tableau 7):

Tableau 7. L'utilisation des pronoms sujets dans la postposition clitique au début et à la fin du XXe siècle dans l'oral mis en scène

\begin{tabular}{|c|c|}
\hline $1900-1908$ & 1985-1997 \\
\hline il (33), elle (6), ils (3), elles (3) $=45$ & $t u(22)$, vous $(12)=34$ \\
\hline vous (31), tu (9) $=40$ & il (9), elle (5), ils (3) $=17$ \\
\hline nous (6) & je (8) \\
\hline je (5) & nous (5) \\
\hline on (3) & on $(1)$ \\
\hline \multicolumn{2}{|l|}{ ce (3) } \\
\hline Total : 102 & Total : 65 \\
\hline
\end{tabular}

L'utilisation, moins importante, des pronoms de troisième personne dans la dernière période paraît, beaucoup mieux que dans la première période, appuyer la constatation de Coveney (2002: 210) selon laquelle, en raison de la resyllabification de la dernière consonne du verbe opérée par le pronom sujet clitique ill(s), elle(s) ou on, l'inversion est alors défavorisée. ${ }^{19} \mathrm{Si}$, pour la première période, les pronoms majoritairement employés pour l'inversion étaient ceux de

\footnotetext{
${ }^{19}$ Coveney (2002: 210) donne l'exemple Cessent-ils pour autant d'être des chômeurs ? (Antenne 2, $5 / 2 / 1988$ ) où le début de l'occurrence avait été interprété par une locutrice native comme " ces styles ».
} 
troisième personne, pour la dernière période, ce sont ceux désignant l'allocutaire, sous forme $t u$ ou vous, qui prédominent. On note désormais (contrairement à la première période) une prépondérance de $t u$ reflétant sans doute (voir cependant 3.3 infra) une plus grande tendance au tutoiement (voir par exemple Coveney 2016).

Bien que le présent soit, pour les deux périodes, le temps majoritaire, les verbes à ce temps se combinent peu (deux fois dans la première période, quatre fois dans la dernière) avec le pronom sujet de première personne. Ces six occurrences n'impliquent pas de verbe du premier groupe et n'incluent que des verbes indiqués dans la liste de Coveney (2002: 190): vais-je, veux-je, suis-je (2), puis-je (2). L'occurrence de veux-je, (1) Où veux-je aller ? (André Gide, L'immoraliste, 1902) ${ }^{20}$ semble toutefois constituer un cas intéressant car, pour Coveney (2002: 191), " the most recent examples of veux-je in any of the sources consulted are [...] both from nineteenth-century literature ».

On terminera cet examen des facteurs linguistiques à l'œuvre, pour la postposition clitique, dans l'oral mis en scène, en insistant sur le fait qu'à de rares exceptions près, tel le cas de veux-je, néanmoins daté du début du $\mathrm{XX}^{\mathrm{e}}$ siècle, non seulement les normes prescriptives sont respectées mais également ce que Houdebine (2015: 16) a appelé les normes communicationnelles, c'est-à-dire les normes subjectives les moins prescriptives, qui " ne s'étayent pas d'un discours de type académique ou grammatical traditionnel, mais plutôt le récusent, au profit d'une écoute des usages, soit des normes statistiques ». En effet, les occurrences de notre extrait de corpus, au-delà de leur caractère grammatical, se plient à d'autres restrictions, de sorte que peu d'entre elles encourent le reproche de bizarrerie ou de " cocasserie ", comme ce serait par exemple le cas pour des formes telles que sont-ce, parlé-je, cours-je, qui, " en dépit de leur respect d'une norme prescriptive, sont ressenties comme étrangères à la langue " (Druetta 2011).

\subsection{Facteurs extralinguistiques}

Pour ce qui est des caractéristiques des personnages et des situations de communication fictionnelles, on remarquera tout d'abord que, de manière plus réaliste, les personnages utilisant cette structure sont en proportion plus éduqués en fin de siècle qu'en début de siècle (respectivement 53 cas sur 65 vs 74 cas sur 102). La structure, alors encore plus favorisée par les normes prescriptives et donc présentée comme non marquée, était associée à une surgénéralisation diastratique la menant à être parfois placée dans la bouche de personnages peu cultivés. La part des situations publiques, d'environ un quart, se maintient mais celle des situations familières augmente, d'environ un tiers à plus de la moitié, avec renforcement en fin de siècle du caractère familier soutenu, peut-être dû à une plus grande représentation de conversations entre amis cultivés dans le corpus. Enfin, la proportion de locuteurs jeunes, de moins de 30 ans, décroît drastiquement de plus du tiers à moins du sixième, reflétant mimétiquement le déclin de la structure également dans l'oral mis en scène.

\footnotetext{
${ }^{20}$ Sauf précision contraire, les exemples sont tirés de Frantext. Seuls sont indiqués le nom de l'auteur, le titre de l'œuvre et la date.
} 


\section{La structure, en augmentation dans l'oral mis en scène, (prép) où $\mathbf{S ~ V ~}$}

On traite, sous la structure (prép) où $\mathrm{S} V$, à la fois (prép) où CL V et (prép) où $\mathrm{N} \mathrm{V}$. Cette dernière sous-structure est demeurée très rare tout au long du $\mathrm{XX}^{\mathrm{e}}$ siècle ${ }^{21}$ (de manière convergente avec l'oral réel, où les sujets nominaux sont peu répandus). En revanche, la sous-structure (prép) où CL V, quasi inexistante dans l'oral mis en scène dans le premier quart du $\mathrm{XX}^{\mathrm{e}}$ siècle, a augmenté en proportion de manière constante, d'une part, et considérable entre le deuxième et le troisième quart, d'autre part (Tableau 8):

Tableau 8. L'évolution quantitative dans l'oral mis en scène au XXe siècle de la structure (prép) où $\mathrm{S} V$

\begin{tabular}{|c|c|c|c|c|}
\hline $\begin{array}{l}\text { Période (date } \\
\text { de l'œuvre) }\end{array}$ & $1900-1925$ & $1926-1950$ & 1951-1975 & 1976-1997 \\
\hline Où CL V & 6 & 32 & 57 & 50 \\
\hline Prép où CL V & 1 & 3 & 9 & 13 \\
\hline Où N V & 1 & 1 & 4 & 1 \\
\hline Prép où N V & & 4 & & \\
\hline Pourcentage & $8 / 649=1,23 \%$ & $40 / 1009=3,96 \%$ & $70 / 642=10,9 \%$ & $64 / 431=14,84 \%$ \\
\hline
\end{tabular}

\section{1. État de l'art}

L'évolution quantitative de la sous-structure (prép) où CL V paraît refléter, certes avec un certain retard, l'avancée de la structure Q S V en français parlé au XX siècle, si l'on en juge par les observations, notamment, de Foulet (1921) et de Fromaigeat (1938):

« [...] n'y aura-t-il pas économie pour [la langue] à dire tout simplement comment tu fais ?, pourquoi elle y va ?. Et en effet, ce sont des phrases qu'on entend aujourd'hui. » (Foulet 1921: 281)

"Dans la langue usuelle familière, une forme [...] avec le mot interrogatif placé au début, mais sans autre moyen syntaxique d'interrogation, semble gagner du terrain. » (Fromaigeat 1938: 40)

La structure Q S V est l'objet de jugements sévères chez Foulet (1921: 324), qui la catégorise comme populaire:

"La langue écrite y voit non plus une bizarrerie sans conséquence, mais une grave incorrection. La langue parlée fait également grise mine à ces tournures, mais elle est loin de leur être aussi réfractaire qu'elle en a l'intention. Elle a subi ici encore l'influence de la langue populaire. »

Une quinzaine d'années plus tard, Fromaigeat (1938: 15) émet un avis plus diversifié:

\footnotetext{
${ }^{21}$ Un exemple de la sous-structure (prép) où $\mathrm{N}$ V est donné en (4). Il s'agit de la seule occurrence du corpus dans le premier quart de siècle.
} 
" Comme cette forme est combattue par les grammairiens, je me contenterai de citer un certain nombre d'exemples pour prouver qu'elle est bien vivante, même dans la bonne société, surtout à Paris et dans les régions où [l'interrogation en est-ce que] ne prédomine pas. "

Fromaigeat (1938: 15 et 16) précise qu'en plus d'être produite par des «Parisiens cultivés ", "il arrive aussi que cette forme serve à évoquer le langage enfantin ou populaire ». La caractéristique populaire, diastratique, semble cependant assimilée à la caractéristique familière, d'ordre diaphasique: « la forme [...] [où il va ?] est encore souvent considérée comme familière ou enfantine » (Fromaigeat 1938: 27).

Dans les enquêtes récentes sur l'oral réel, Coveney (2002: 228) signale une forte tendance à recourir à la structure Q S V quand l'interrogatif est comment (19 occurrences sur 25 , soit $76 \%$ ), en raison de l'existence de séquences plus ou moins figées telles que comment $X$ s'appelle ?, mais aussi, dans une moindre mesure, quand l'interrogatif est où (5 occurrences sur 11, soit 45,5\%). Dans le corpus de Druetta (2009: 53-55), sur les 22 occurrences en où, 3 se conforment à cette structure.

\subsection{Facteurs linguistiques}

On commentera plus particulièrement, pour cette structure, les 8 occurrences produites entre 1900 et 1925 et les 64 occurrences produites entre 1976 et 1997. Druetta (2009: 159) note que les proformes (adverbes ou pronoms) régies mais n'entrant pas dans la valence du verbe, ${ }^{22}$ de par leur « lien moins étroit » avec celui-ci, ont une plus grande latitude positionnelle et peuvent notamment apparaître plus facilement à l'initiale. Néanmoins, dans notre extrait de corpus, où, bien qu'à l'initiale, fait le plus souvent partie de la valence verbale (6 cas dans le premier quart de siècle et 44 cas dans le dernier quart de siècle), sans doute en raison de la fréquence des verbes à valence locative, tels être et aller:

(2) Mais où tu vas comme cela, mon chéri ? (Marcel Proust, La recherche $16,1922)$

(3) Et ta 1000 ? Où elle est? (Alain Page, Tchao pantin, 1982)

Coveney (2002: 227-228) remarque, dans son corpus d'oral réel, peu de restrictions d'ordre linguistique ou d'ordre pragmatique pour la structure Q S V, si ce n'est, peut-être, qu'elle " tends to be avoided for emphatic assertions (rhetorical questions) ». Cette constatation ne vaut pas pour notre extrait de corpus, où l'on compte une assez forte minorité de questions rhétoriques, ${ }^{23} 2$ occurrences pour le premier quart de siècle et 12 pour le dernier quart de siècle:

\footnotetext{
${ }^{22}$ Druetta (2009: 159) précise que, " pour où, il s'agit des valences locatives de verbes comme être, se trouver, aller, passer, se rendre, etc. ». Dans ce dernier cas, où peut être analysé, non comme un complément simplement régi, mais comme ce qui est parfois appelé un complément de verbe.

${ }^{23}$ Nous adoptons ici la définition de Riegel et al. (2009: 561), selon laquelle « une interrogation oratoire ne constitue pas une question ouverte, mais est dirigée vers une réponse positive ou négative ».
} 
(4) - [...] Sur celle qui n'a pas de rubis il y a bien un aigle, mais sur l'autre c'est une espèce de tête d'homme qui est ciselée.- Une tête d'homme? Où monsieur a vu ça? Rien qu'avec mes lorgnons j'ai tout de suite vu que c'était une des ailes de l'aigle [...]. (Marcel Proust, La recherche 20, 1922)

(5) Non mais, où tu te crois? (Gérard Mordillat, Vive la sociale, 1981)

\subsection{Facteurs extralinguistiques}

Pour terminer, on évoquera quelques facteurs " externes " relatifs à la structure (prép) où $\mathrm{S} \mathrm{V}$ dans l'oral mis en scène (Tableau 9):

Tableau 9. Caractéristiques des personnages et des situations de communication fictionnelles pour la structure (prép) où S V au début et à la fin du XXe siècle dans l'oral mis en scène*

\begin{tabular}{|c|c|c|c|c|}
\hline \multirow[b]{2}{*}{ Caractéristiques } & \multicolumn{3}{|c|}{ des personnages } & \multirow{2}{*}{$\begin{array}{c}\text { des situations de } \\
\text { communication } \\
\text { fictionnelles } \\
\text { Familier }\end{array}$} \\
\hline & Enfant ou adolescent & Populaire & $\begin{array}{l}\text { Personnage } \\
\text { masculin }\end{array}$ & \\
\hline $\begin{array}{l}1900-1925 \\
8 \text { occurrences }\end{array}$ & 3 & 3 & 3 & $\begin{array}{c}1 \\
\text { (familier bourgeois) }\end{array}$ \\
\hline $\begin{array}{l}1976-1997 \\
64 \text { occurrences }\end{array}$ & $\begin{array}{c}19 \\
(+11 \text { questions posées à } \\
\text { des enfants ou adolescents) }\end{array}$ & 29 & 34 & 52 \\
\hline
\end{tabular}

*Les catégories ne sont pas mutuellement exclusives. Le total des lignes peut donc être supérieur au nombre d'occurrences.

On se souvient que la structure Q S V, dans les études de Behnstedt (1973) et de Söll (1982), figurait surtout "dans les trois corpus de langue spontanée » (Coveney 2011: 127), en première place dans les variétés de bourgeois familier et d'ouvriers de Behnstedt (1973) et en deuxième place chez les enfants de Söll (1982). Dans notre extrait de corpus, ces tendances sont particulièrement vérifiées dans le dernier quart de siècle, avant tout d'un point de vue diaphasique, le registre familier caractérisant l'immense majorité des occurrences (sans, toutefois, qu'il puisse être établi qu'il s'agit de familier bourgeois), et, dans une moindre mesure, d'un point de vue diastratique, presque la moitié des occurrences émanant de personnages issus des couches populaires. Si les occurrences relèvent également de personnages enfants ou adolescents, à hauteur de presque un tiers, un autre paramètre paraît jouer: les caractéristiques des personnages interlocuteurs, plus d'un sixième de ces questions étant adressé à des enfants ou des adolescents. Enfin, conformément à la constatation de Coveney (2002: 247-248) pour l'oral réel, on trouve une majorité de personnages locuteurs masculins utilisant cette structure dans le dernier quart de siècle, ce qui est généralement relié (cf. Trudgill 1972) à la tendance des hommes à recourir à des traits plus vernaculaires, moins standard, que les femmes (cependant, on note une prépondérance générale, dans l'oral mis en scène, de personnages locuteurs masculins, tout au moins posant des questions fondées sur où). 


\section{La structure, en augmentation dans l'oral mis en scène, S V (prép) où (dite in situ)}

La structure S V (prép) où, quasi inexistante dans l'oral mis en scène dans la première moitié du $\mathrm{XX}^{\mathrm{e}}$ siècle, a augmenté de manière considérable dans la seconde moitié, tout particulièrement dans le dernier quart (Tableau 10). Il convient néanmoins, à l'intérieur de cette structure, de distinguer la sousstructure avec sujet non clitique, pratiquement absente, tout au long du siècle, de l'oral mis en scène (en cohérence avec l'oral réel où les sujets nominaux sont peu répandus, comme il a déjà été noté).

Tableau 10. L'évolution quantitative dans l'oral mis en scène au XXe siècle de la structure S V (prép) où

\begin{tabular}{|c|c|c|c|c|}
\hline $\begin{array}{c}\text { Période (date } \\
\text { de l'œuvre) }\end{array}$ & $1900-1925$ & $1926-1950$ & $1951-1975$ & 1976-1997 \\
\hline CL V prép où & & 2 & 4 & 6 \\
\hline $\mathrm{CL} V$ où & 1 & 3 & 25 & 57 \\
\hline N V où & 1 & & & 1 \\
\hline N V prép où & 1 & 1 & 1 & \\
\hline Pourcentage & $3 / 649=0,46 \%$ & $6 / 1009=0,59 \%$ & $30 / 642=4,67 \%$ & $64 / 431=14,84 \%$ \\
\hline
\end{tabular}

\section{1. État de l'art}

L'évolution quantitative de la structure $S \mathrm{~V}$ (prép) où dans l'oral mis en scène paraît refléter l'avancée de la structure $\mathrm{S} V \mathrm{Q}$ dans l'oral réel au XX $\mathrm{X}^{\mathrm{e}}$ siècle. Selon Coveney (2011: 125), « on trouve peu de traces de [cette] structure [...] avant la fin du XIX ${ }^{\mathrm{e}}$ siècle ». De manière convergente, si Larrivée (2019a: 107) signale que " la construction s'installe [au XVIII ${ }^{\mathrm{e}}$ siècle], et dans l'oral représenté de textes littéraires ", il n'en indique toutefois qu'un très faible pourcentage avant $1900^{24}$. Pour Foulet (1921: 323), si " ces phrases ne pénètrent guère dans les livres ", elles sont "très fréquentes et semblent gagner du terrain ». Fromaigeat (1938: 16-17) ajoute, une quinzaine d'années plus tard, que "l'interrogation avec le mot interrogatif placé à la fin » est " courante ", " pour faire répéter une partie de la phrase ", tout en précisant :

«[...] lorsqu'il s'agit de solliciter un complément non encore énoncé de la proposition, cette forme interrogative est très combattue par les puristes, quoiqu'on ne puisse pas du tout la considérer comme caractéristique du français populaire et négligé. La conversation des personnes cultivées en offre de nombreux exemples dans toutes les régions de langue française. »

Coveney (2011: 125) note que Tuaillon (1975) « appelle [la structure S V Q] 'le dernier-né" des tours interrogatifs " et que, pour celui-ci, "cette variante s'est répandue seulement après 1945 ». Larrivée (2019b: 119) remarque d'ailleurs, pour l'interrogatif comment, une forte augmentation des interrogatives in situ,

\footnotetext{
${ }^{24}$ Selon Larrivée (2019a: 105), pour l'ensemble des trois interrogatifs étudiés, qui, comment et combien, dans Frantext, entre 1700 et 1900, les interrogatives in situ sont au nombre de 7, pour un total de 2932.
} 
dans des entretiens semi-dirigés, dans le corpus ESLO2 (2014), soit 70 in situ sur un total de 1113 interrogatives, par rapport au corpus ESLO1 (1969-1974), ${ }^{25}$ soit 13 sur 1434. De nos jours, pour Coveney (2011: 125), cette structure, privilégiée par des locuteurs jeunes, " est en train de gagner du terrain en français familier "; Dagnac (2014) souligne que les interrogatives comportant cette structure " ne sont pas stigmatisées ».

Sur un plan linguistique, Druetta (2011) mentionne l'impossibilité, pour ces constructions où l'interrogatif est placé à la fin, de commencer par est-ce que, ce qui signalerait une interrogative totale, de manière contradictoire avec la présence d'une proforme $q u$ - indiquant une interrogative partielle.

Coveney (2002: 220), conformément à Behnstedt (1973: 93), observe que certaines proformes favorisent la structure S V Q, telles que combien (notamment pour des raisons de longueur, combien étant souvent suivi d'un nom précédé de la préposition de), à l'inverse d'autres proformes telles que pourquoi, comment et où. Toutefois, l'enquête de Druetta (2009) montre, au contraire, aux côtés de combien, une préférence de où pour la position in situ ( $74 \%$ d'interrogatives in situ pour où et $57 \%$ pour combien). Nous ne pouvons nous prononcer à cet égard ni dans un sens ni dans l'autre pour l'oral mis en scène, notre étude étant axée sur où. Quoi qu'il en soit, la nature même de l'interrogatif n'a pas empêché une forte tendance à l'augmentation de la structure in situ dans l'oral représenté dans la seconde moitié du $\mathrm{XX}^{\mathrm{e}}$ siècle.

\subsection{Facteurs linguistiques}

On commentera plus particulièrement, pour cette structure, les 3 occurrences produites entre 1900 et 1925 et les 64 occurrences produites entre 1976 et 1997. Comme indiqué supra en 4.2, Druetta (2009: 159) note que les proformes entrant dans la valence du verbe " manifestent une préférence pour la distribution postverbale ", ce qui, selon lui, favorise où (et combien) dans la position in situ. Dans notre extrait de corpus, où placé en finale fait en effet très souvent partie de la valence verbale ( 2 cas sur 3 dans le premier quart de siècle et 57 cas sur 64 dans le dernier quart de siècle). On se souvient néanmoins que, dans la structure (prép) où $\mathrm{S} \mathrm{V}$, où faisait aussi très majoritairement partie (bien qu'à moindre hauteur) de la valence verbale ( 6 cas sur 8 dans le premier quart de siècle et 44 cas sur 64 dans le dernier quart de siècle).

La très forte tendance d'un où valenciel dans la position in situ peut être reliée à une encore plus grande fréquence du verbe être exprimant une localisation statique, dont on compte 20 occurrences (sur 64) pour le dernier quart de siècle. Cette constatation, alliée à celle d'une forte propension, générale, à des sujets pronominaux dans les interrogatives, appuie l'hypothèse, déjà vérifiée dans Coveney (1995) pour l'oral réel, selon laquelle la partie " sujet-verbecomplément" (hors interrogatif) de l'interrogative in situ est peu informative, d'autant que, lorsque l'interrogatif est où, la partie "sujet-verbe-complément " se réduit souvent à une partie "sujet-verbe ». En effet, si l'on trouve un complément (autre que où) dans 2 cas sur 3 dans le premier quart de siècle, la proportion tombe à 18 cas sur 64 dans le dernier quart de siècle.

${ }^{25}$ ESLO, Enquêtes SocioLinguistiques à Orléans, http://eslo.huma-num.fr/ 
Sur un plan plus pragmatique, on signalera l'absence totale d'interrogatives in situ négatives dans notre extrait de corpus, conformément à ce que note Coveney (2011: 132-133). Par ailleurs, Coveney (1995) souligne que les interrogatives in situ seraient moins utilisées lorsque le locuteur n'attend pas de réponse. Cette tendance se vérifie pour l'oral mis en scène, les occurrences dans notre extrait de corpus comportant majoritairement des réponses: 3 cas sur 3 dans le premier quart de siècle, 50 cas sur 64 dans le dernier quart de siècle. Obenauer (1994), selon Coveney (2011: 136), « considérait que S V Q est acceptable uniquement dans des contextes où la réponse peut être jugée importante [et que,] par conséquent, cette structure serait exclue des questions rhétoriques (où la réponse est supposée connue de l'interlocuteur) ». Il convient cependant de souligner que quelques réponses ( 2 dans le premier quart de siècle et 1 dans le dernier quart de siècle) sont des autoréponses, ce qui conduit à voir dans les exemples suivants respectivement une question introductive (l'interrogative " introdui[sant] l'assertion suivante, afin de la mettre en relief » (Dekhissi et Coveney 2018: 129)) et une demande de confirmation:

(6) Je vous prie d'éviter avec égoïsme aucune émotion, et je vous trouve où ? En pleine forêt vierge de la Guyanne, en butte aux pires aventures de voyage! (Louis Delattre, Carnets d'un médecin de village, 1910)

(7) Tu la trouves où ta thune ? Prosper est sorti de zonzon et y t'saute? (Denis Belloc, Kepas, 1989)

Dans un autre cas, à la fois le contexte antérieur et le contenu de la réponse font pencher pour une question rhétorique (le tien est où ? pouvant être interprété comme «le tien n'est pas là ») :

(8) Où sont tous les maris? ai-je demandé, finalement.

- Le mien est là, apparemment. Et toi, le tien est où ? a répliqué méchamment Martha, comme si j'agressais sa famille.

- Tu le sais mieux que moi! ai-je répondu tristement. (Geneviève Brisac, Week-end de chasse à la mère, 1996)

Cette occurrence vient à l'appui de Coveney (2011: 140) qui, s'il n'a " pas trouvé d'exemples réels de [structure] S V Q employée comme question rhétorique, [pense que] cette possibilité ne semble pas totalement exclue ». Néanmoins, dans notre extrait de corpus, les interrogatives peuvent presque toutes être interprétées comme des demandes d'information. On remarque aussi, parmi ces demandes d'information, 4 questions échos dans le dernier quart de siècle. Ainsi, dans l'occurrence suivante, le personnage locuteur répète une partie de la question du tour de parole précédent tout en demandant clarification pour l'élément (l'adverbe $y$ ) auquel se substitue l'interrogatif in situ:

(9) - Bon, dit Flesch, on y va.

- On va où?

- À la morgue, nom de Dieu! (Anne Vergne, L'innocence du boucher, 1984) 
Comme indiqué supra en 5.1, Fromaigeat (1938:16) notait, pour « l'interrogation avec le mot interrogatif placé à la fin », que, "pour faire répéter une partie de la phrase, cette sorte de question est toute naturelle ». Cependant, la part des questions échos est très minoritaire, conformément à ce qui se produit, tout au moins dans la période récente, dans l'oral réel, selon Coveney (2002: 218): «In French it is certainly the case that the S V Q structure is far more frequent in non-echo contexts ».

\subsection{Facteurs extralinguistiques}

Coveney (2002: 226) émet l'hypothèse que:

" one reason for S V Q being considered more appropriate in colloquial styles, may be that in such contexts, where interlocutors tend to be socially intimate and therefore to share a good deal of background knowledge, the 'presupposition pool' is larger - i.e. more can be taken for granted. »

Dans notre extrait de corpus, l'hypothèse paraît vérifiée, non pour le premier quart de siècle, où au moins 2 cas sur 3 relèvent d'une situation relativement formelle (le nombre d'occurrences étant de toute façon trop faible), mais pour le dernier quart de siècle, où 54 cas sur 64 s'inscrivent dans une situation familière. Coveney (2011: 125) ajoute que, "dans une étude récente d'un corpus oral important, Quillard (2000) a trouvé que [la structure] S V Q est utilisée davantage par les locuteurs âgés de moins de 35 ans ». Dans notre extrait de corpus, les personnages locuteurs sont dans 20 cas (sur 64) des enfants ou de jeunes adultes pour le dernier quart de siècle (dans 1 cas (sur 3) pour le premier quart de siècle). Enfin, on compte 2 personnages locuteurs nettement éduqués pour le premier quart de siècle et 22 pour le dernier quart, ce qui confirme que les interrogatives comportant cette structure "ne sont pas stigmatisées " (Dagnac 2014), même dans l'oral mis en scène. D'une certaine façon, la structure S V (prép) où apparaît en concurrence avec la structure (prép) où S V, qui, dans une immense majorité des cas, est également associée à un registre familier. On remarque d'ailleurs la ventilation sur ces deux structures de plusieurs personnages, notamment des enfants ou des jeunes des banlieues, ce qui revient, malgré tout, à diminuer la place de la dimension diastratique. À l'appui de cette minoration du diastratique, soulignons aussi que les interrogatives de ces deux structures sont presque toujours associées à une " oralité normée " ou une "oralité courante ", mais non à une "oralité marquée » (Rouayrenc 2010: 249-250) (voir 6.3 infra).

\section{Les structures, en diminution dans l'oral mis en scène, débutant par (prép) où que}

Dans l'oral mis en scène, les structures interrogatives débutant par (prép) où que accusent une diminution constante en proportion (Tableau 11), en cohérence avec l'oral réel, où la structure Q que $\mathrm{S} \mathrm{V}$ est signalée comme étant en déclin (voir par exemple Dagnac 2014). En particulier, la baisse est drastique vers le milieu du $\mathrm{XX}^{\mathrm{e}}$ siècle, ce qui paraît refléter, ici de manière non différée, 
l'évolution de Q que S V dans l'oral réel. En effet, comme indiqué supra en 2.1, cette structure est produite dans une assez grande mesure par les ouvriers et dans une faible mesure par les enfants, selon les enquêtes respectives de Behnstedt (1973) et de Söll (1982). Elle est en revanche absente dans les enquêtes de Coveney (1996/2002) et de Druetta (2009).

Tableau 11. L'évolution quantitative dans l'oral mis en scène au XXe siècle des structures débutant par (prép) où que

\begin{tabular}{|c|c|c|c|c|}
\hline $\begin{array}{ll} & \text { Période (date } \\
\text { Type de l'œuvre) }\end{array}$ & 1900-1925 & $1926-1950$ & $1951-1975$ & 1976-1997 \\
\hline Où que CL V & 40 & 48 & 11 & 6 \\
\hline Prép où que CL V & 12 & 12 & 2 & 1 \\
\hline Où que N V & 1 & 1 & & \\
\hline Où que V N & 11 & 8 & 4 & 1 \\
\hline Où qu'il y a N & & 1 & & \\
\hline Où que sans V & 1 & & & 1 \\
\hline Où que c'est que CL V & & 1 & & 1 \\
\hline Pourcentage & $65 / 649=10,01 \%$ & $71 / 1009=7,03 \%$ & $17 / 642=2,64 \%$ & $10 / 431=2,32 \%$ \\
\hline
\end{tabular}

\section{1. État de l'art}

Pour Foulet (1921: 321), qui donne l'exemple où que tu vas ?, il s'agit de l'" une des tournures essentielles de la langue populaire », " emploi nettement fautif [...] qui tend à se généraliser ". Sur un plan diatopique, Foulet (1921: 321) précise que cette construction est " courante à Paris et sur presque tout le domaine de la langue d'oïl ». D’après Behnstedt (1973: 36), cité par Coveney (2002: 101), la structure relève aussi plus des variétés du nord que de celles du sud.

Pour ce qui est de la catégorisation de que, Foulet (1921:272) évoque une «particule» qu'il rapproche de est-ce que, les deux « détach[ant] avec la même netteté le pronom ou l'adverbe initial qui porte tout l'essentiel de l'interrogation: Où est-ce que tu vas ?, où que tu vas ? ", que " ayant [toutefois] l'avantage de la brièveté ". Ce rapprochement a par la suite été mené notamment par la grammaire générative qui analyse à la fois est-ce que et que comme des complémenteurs (voir cependant Hadermann (1993: 113-114), qui rapporte une autre hypothèse en concurrence, posant que " où que et où est-ce que ne sont pas apparentés »). Druetta (2003: 31), en l'absence d'éléments suffisants, ne souhaite pas se prononcer "sur la nature du marquage interrogatif par que seul ". Druetta (2011) ajoute que que " réalise une segmentation de l'énoncé comparable à celle mise en place par le clivage " mais que, pour autant, " il n'est pas nécessaire de postuler la réduction d'une structure de clivage pour rendre compte de ce fonctionnement». 


\subsection{Facteurs linguistiques}

On commentera plus particulièrement, pour ces structures, les 65 occurrences produites entre 1900 et 1925 et les 10 occurrences produites entre 1976 et 1997. Dans notre extrait de corpus, une forme, mentionnée par Foulet (1921: 282), n'apparaît jamais en tant qu'interrogative directe : il s'agit de où (suivi de que) précédé de là, du type là où qu’il est ? (exemple de Foulet (1921: 282)), dans la mesure où «où est une forme très courte qui offre moins de prise que combien, comment, pourquoi. On la renforce donc parfois à l'aide de là ». On notera en revanche, montrant sans doute ce même souci de renforcement, la présence de 5 occurrences du type où ça que CL V, uniquement dans la première moitié du siècle (2 entre 1900 et 1925, 3 entre 1926 et 1950): (10) où ça qu'ils arrivent? (René Benjamin, Gaspard, 1915).

La structure où que $\mathrm{V} \mathrm{N}$, bien que toujours fortement minoritaire, se situe en deuxième place après la structure (prép) où que $\mathrm{CL} \mathrm{V}$ dans les trois premiers quarts de siècle (mais on n'en trouve qu'une occurrence dans le dernier quart de siècle): (11) où qu'est l'ambulance ? (Roland Dorgelès, Les croix de bois, 1919). Comme il a été signalé supra en 2.1 , les enquêtes sur le français parlé ne mettent pas toujours en évidence la présence de structures en que. Lorsqu'elles le font, ces structures ne sont pas détaillées, ce qui rend difficile une comparaison avec l'oral mis en scène. Néanmoins, on peut penser que la structure où que $\mathrm{V} \mathrm{N}$, dans l'oral réel, est d'autant plus rare que la structure " simple " à postposition nominale, où $\mathrm{V} \mathrm{N}$, est déjà faiblement répandue en langue parlée, où les sujets nominaux sont peu fréquents. On aurait donc ici affaire, en quelque sorte, à une invention de l'oral représenté (nettement moins exploitée dans le dernier quart de siècle), allant à l'encontre de la remarque de Foulet (1921: 273) : " si la langue populaire a accueilli ces tournures [notamment en que] avec tant de faveur, c'est parce qu'elles lui fournissaient le moyen de se débarrasser d'inversions gênantes ». Le fait que cette remarque ne s'applique pas à cette construction peut être imputé au côté hybride de cette dernière, à la fois de conception écrite et de registre populaire. Sa quasi-disparition va également dans le sens d'un réalisme accru.

\subsection{Facteurs extralinguistiques}

Pour ce qui concerne les caractéristiques des personnages et des situations de communication fictionnelles, on note, tout d'abord, pour le premier quart de siècle, que la plupart des interrogatives sont placées dans la bouche de personnages, populaires, "récurrents ", le personnage le plus " productif " étant Gaspard du roman éponyme de René Benjamin (1915), à l'origine de 20 interrogatives en où que dans notre corpus. Ce phénomène (presque absent dans le dernier quart de siècle, notamment du fait de la rareté des occurrences) entraîne une fixation diastratique, pouvant aussi être vue comme une minimisation de la part du diaphasique. Les dialogues sont ainsi utilisés «moins [dans] une fonction dramatique [...] que [dans] une fonction analytique, caractérisante, visant à décrire [les personnages] par leur idiolecte " (Denoyelle 2018: 206). On remarquera en outre que ces idiolectes sont généralement liés à 
une « oralité marquée » (Rouayrenc 2010: 250), qui se manifeste, en particulier, par " des suppressions de lettres »: (12) d'où qu'vous venez? D’où qu'vous êtes? (René Benjamin, Gaspard, 1915). Sur le plan diatopique, à part dans une dizaine de cas indéterminés pour le début du siècle et un cas indéterminé pour la fin du siècle, les interrogatives sont émises par des personnages du nord de la France, en conformité avec Behnstedt (1973: 36), cité par Coveney (2002: 101), selon lequel la structure relève plus des variétés du nord que de celles du sud (voir 6.1 supra). De manière écrasante, les personnages locuteurs sont masculins, qu'il s'agisse du premier quart de siècle (57 cas sur 65 , plus un cas indéterminé) ou du dernier ( 9 cas sur 10): est ici mimée très nettement la tendance des hommes, dans l'oral réel, à recourir à des traits plus vernaculaires, moins standard, que les femmes.

L'exemple suivant du début $\mathrm{du} \mathrm{XX}^{\mathrm{e}}$ siècle est particulièrement intéressant car l'interrogative en que apparaît dans un polylogue ${ }^{26}$ organisé en "liste paradigmatique ", où " les répliques [...] sont montées non 'en série' mais en 'en parallèle': elles ne s'enchaînent pas les unes aux autres mais sont juxtaposées, sans hiérarchie apparente " (Denoyelle 2018: 201). Ce procédé est utilisé "à des fins de caractérisation, non des personnages individuellement mais du groupe » (Denoyelle 2018: 205), d'autant plus que l'ensemble du polylogue est ici introduit par un seul segment citant:

(13) Tassés le long du fossé, des régiments arrêtés nous regardaient passer. [...] D'eux à nous, des voix se hélaient :

- Quel régiment?

- Ce qu'il y a encore un patelin avant les tranchées?

- D'où que vous venez? (Roland Dorgelès, Les croix de bois, 1919)

\section{Conclusion}

Foulet (1921: 261) déclarait: "Si la littérature n'accueille qu'avec défiance les tournures nouvelles, elle conserve jalousement les archaïsmes, elle est toujours très en retard sur le développement de la langue ". Néanmoins, comme a contribué à le montrer cette étude, même la littérature évolue. Les structures interrogatives, ici fondées sur où, n'ont pas coexisté de manière stable dans l'oral mis en scène du $\mathrm{XX}^{\mathrm{e}}$ siècle qui révèle, certes de manière atténuée et différée (sauf, semble-t-il, dans le cas des structures débutant par (prép) où que), ce qui vaut pour l'oral réel: d'un côté, le déclin de la postposition du sujet pronominal clitique et des structures en que; de l'autre, l'essor des structures (prép) Q S V et S V (prép) $\mathrm{Q}^{27}$ (Tableau 12).

\footnotetext{
${ }^{26}$ Les polylogues peuvent être définis comme des " conversations à plusieurs participants ", beaucoup "plus fréquentes " dans la vie réelle que les conversations à deux, en revanche " plus rares " dans la littérature (Denoyelle 2018: 199).

${ }^{27}$ Signalons que des tendances semblables, déclin de la postposition du sujet pronominal clitique et essor d'abord de la structure Q S V puis de la structure in situ, ont été mises au jour dans l'étude de Farmer (2015) portant sur les dialogues de 60 films de 1930 à 2009 (voir aussi Dekhissi 2013, Dekhissi et Coveney 2018). Si les tendances sont comparables avec celles de l'oral mis en scène dans les dialogues de romans, ce n'est toutefois pas le cas pour les proportions puisque, entre 1930 et 2009, l'inversion pronominale (y
} 
Tableau 12. Récapitulation de l'évolution quantitative dans l'oral mis en scène au XXe siècle des structures (prép) où V CL, (prép) où S V, S V (prép) où et débutant par (prép) où que

\begin{tabular}{|c|c|c|c|c|c|}
\hline Type & $\begin{array}{l}\text { Période } \\
\text { (date de } \\
\text { l'œuvre) }\end{array}$ & $\begin{array}{c}1900-1925 \\
649 \text { occurrences }\end{array}$ & $\begin{array}{c}1926-1950 \\
1009 \text { occurrences }\end{array}$ & $\begin{array}{c}1951-1975 \\
642 \text { occurrences }\end{array}$ & $\begin{array}{c}1976-1997 \\
431 \text { occurrences }\end{array}$ \\
\hline \multicolumn{2}{|c|}{ (Prép) où V CL } & $\begin{array}{l}356 \text { occurrences } \\
=54,85 \%\end{array}$ & $\begin{array}{l}538 \text { occurrences } \\
\quad=53,32 \%\end{array}$ & $\begin{array}{c}316 \text { occurrences } \\
=49,22 \%\end{array}$ & $\begin{array}{c}156 \text { occurrences } \\
=36,19 \%\end{array}$ \\
\hline \multicolumn{2}{|c|}{ (Prép) où S V } & $\begin{array}{c}8 \text { occurrences } \\
=1,23 \%\end{array}$ & $\begin{array}{c}40 \text { occurrences } \\
=3,96 \%\end{array}$ & $\begin{array}{c}70 \text { occurrences } \\
=10,9 \%\end{array}$ & $\begin{array}{l}64 \text { occurrences } \\
=14,84 \%\end{array}$ \\
\hline \multicolumn{2}{|c|}{ S V (prép) où } & $\begin{array}{c}3 \text { occurrences } \\
=0,46 \%\end{array}$ & $\begin{array}{c}6 \text { occurrences } \\
=0,59 \%\end{array}$ & $\begin{array}{c}30 \text { occurrences } \\
=4,67 \%\end{array}$ & $\begin{array}{l}64 \text { occurrences } \\
=14,84 \%\end{array}$ \\
\hline \multicolumn{2}{|c|}{ (Prép) où que } & $\begin{array}{l}65 \text { occurrences } \\
=10,01 \%\end{array}$ & $\begin{array}{l}71 \text { occurrences } \\
=7,03 \%\end{array}$ & $\begin{array}{l}17 \text { occurrences } \\
=2,64 \%\end{array}$ & $\begin{array}{c}10 \text { occurrences } \\
=2,32 \%\end{array}$ \\
\hline
\end{tabular}

A ainsi pu être mise en évidence, au service d'un réalisme accru, une plus grande congruence entre normes objectives de l'oral réel et normes objectives de l'oral mis en scène, certainement liée également au rôle moins important que semblent jouer les normes subjectives prescriptives en fin de $\mathrm{XX}^{\mathrm{e}}$ siècle.

L'étude a en outre souligné une tendance, toujours en fin de $\mathrm{XX}^{\mathrm{e}}$ siècle, à une exploitation de la diversité des structures interrogatives fondées sur où à un niveau plus diaphasique que diastratique. En effet, même si la cohérence diastratique de la structure à postposition clitique s'est renforcée (cette structure étant de moins en moins la structure de prestige, "par défaut "), à la fois la quasi-disparition des structures en que, très stigmatisées diastratiquement, et l'expansion de deux structures concurrentes (prép) où S V et S V (prép) où, à marquage avant tout diaphasique, ont participé à une plus grande prise en compte (mise en scène ?) de cette dernière dimension.

Cette étude sera à compléter par un examen, d'une part, de la structure à postposition nominale, privilégiée dans l'oral représenté, et des structures averbales, ainsi que, d'autre part, des structures " rares » dans l'oral mis en scène dans les dialogues de romans, telles que l'inversion complexe, ou celles centrées sur un verbe non conjugué, ou encore celles en est-ce que. Il conviendra également d'étudier le fonctionnement, dans cet oral mis en scène, de structures interrogatives directes partielles fondées sur d'autres interrogatifs (par exemple, pourquoi, quand, etc.), pour vérifier que des tendances similaires existent. Enfin, il sera intéressant d'établir de manière approfondie des comparaisons avec d'autres sortes d'oraux mis en scène ayant bénéficié d'études avec un éclairage diachronique, tels les dialogues de films (Dekhissi 2013, Dekhissi et Coveney 2018, Farmer 2015, Jansen et al. 2020). 


\section{Références}

Behnstedt, P. (1973). Viens-tu ? Est-ce que tu viens? Tu viens ? Formen und Strukturen des direkten Fragesatzes im Französischen. Tübingen: Narr.

Byrne, L. S. R. et Churchill, E. L. (1986). A Comprehensive French Grammar. Oxford: Blackwell (3e édition révisée par G. Price).

Coveney, A. (1995). The use of the QU-final interrogative structure in spoken French. Journal of French Language Studies, 5: 143-171.

Coveney, A. (2002). Variability in Spoken French: A Sociolinguistic Study of Interrogation and Negation. Bristol: Elm Bank.

Coveney, A. (2011). L'interrogation directe. Travaux de linguistique, 63.2: 112-145.

Coveney, A. (2016). La quête du vernaculaire dans l'étude de la variation grammaticale. $5^{\text {ème }}$ Congrès Mondial de Linguistique Française - CMLF 2016. Paris: Institut de Linguistique Française, 1-13.

Dagnac, A. (2014). La variation des interrogatives en français. Document préparatoire (texte provisoire) pour contribution à la Grande Grammaire du Français. https://hal.archivesouvertes.fr/hal-00988751

Dekhissi, L. (2013). Variation syntaxique dans le français multiculturel du cinéma de banlieue. Thèse de doctorat, Université d'Exeter.

Dekhissi, L. et Coveney, A. (2018). La variation dans l'emploi des interrogatives partielles dans le cinéma de banlieue. Dans: M.-J. Béguelin, A. Coveney et A. Guryev (éds), L’interrogative en français. Berne: Peter Lang, 119-151.

Denoyelle, C. (2018). Étude diachronique des polylogues littéraires. Dans : H. Barthelmebs-Raguin, G. Komur-Thilloy, J. M. Lopez-Muñoz, S. Marnette et L. Rosier (éds), Le Discours rapporté. Temporalité, histoire, mémoire et patrimoine discursif. Paris: Classiques Garnier, 199-213.

Dewaele, J.-M. (1999). Word order variation in French interrogative structures. ITL Review of Applied Linguistics, 125: 161-180.

Druetta, R. (2003). Qu'est-ce tu fais? État d'avancement de la grammaticalisation de est-ce que. Deuxième partie. Linguae \&: Rivista di lingue e culture moderne, 1: 21-35.

Druetta, R. (2009). La question en français parlé: étude distributionnelle. Turin: Trauben Edizioni.

Druetta, R. (2011). Les formes interrogatives au début du $\mathrm{XXI}^{\mathrm{e}}$ siècle: évolution ou continuité ? L'information grammaticale, 129: version non paginée.

Elsig, M. (2009). Grammatical Variation across Space and Time: The French Interrogative System. Amsterdam/Philadelphie: John Benjamins Publishing Company.

Farmer, K. L. (2015). Sociopragmatic variation in yes/no and wh-interrogatives in hexagonal French: A realtime study of French films from 1930 to 2009. Thèse de doctorat, Université de l'Indiana.

Foulet, L. (1921). Comment ont évolué les formes de l'interrogation. Romania, 47.186-187: 243-348.

Fromaigeat, E. (1938). Les formes de l'interrogation en français moderne : leur emploi, leurs significations et leur valeur stylistique. Vox Romanica, 3: 1-47.

Gadet, F. (1997). La variation, plus qu'une écume. Langue française, 115: 5-18.

Grevisse, M. (1986) et (1993). Le bon usage. Gembloux: Duculot $\left(12^{\mathrm{e}}\right.$ et $13^{\mathrm{e}}$ éditions refondues par A. Goosse).

Guillot-Barbance, C., Pincemin, B. et Lavrentiev, A. (2017). Représentation de l'oral en français médiéval et genres textuels. Langages, 208: 53-68.

Guryev, A. (2017). La forme des interrogatives dans le Corpus suisse de SMS en français: étude multidimensionnelle. Thèse de doctorat, Université de Neuchâtel et Université Paris 3 Sorbonne Nouvelle.

Hadermann, P. (1993). Étude morphosyntaxique du mot où. Paris/Louvain-la-Neuve: Duculot.

Houdebine, A.-M. (2015). De l'imaginaire linguistique à l'imaginaire culturel. La linguistique, 51.1: 3-40.

Jansen, L., Gagsteiger, D. et Pustka, E. (2020). L'interrogation directe dans la version française de trois classiques d'animation de Disney : Blanche Neige (1937), Les Aristochats (1970) et La Reine des Neiges (2013). Dans: J. Hafner, S. Postlep et E. Pustka (éds), Changement et stabilité. La langue française dans les médias audiovisuels du XIX ème au XXI'me siècle. Vienne: LIT, 241-271.

Kerbrat-Orecchioni, C. (1992). Les interactions verbales. 2. Paris: Armand Colin.

Larrivée, P. (2019a). Contextes promoteurs et émergence des questions in situ en français. Dans: A. Dufter, K. Grübl et T. Scharinger (éds), Des parlers d'oül à la francophonie : contact, variation et changement linguistiques. Berlin: De Gruyter, 97-115. 
Larrivée, P. (2019b). Historical pragmatics, explicit activation and wh in situ in French. Dans: I. Feldhausen, M. Elsig, I. Kuchenbrandt et M. Neuhaus (éds), Romance Languages and Linguistic Theory 15: Selected papers from 'Going Romance' 30, Frankfurt. Amsterdam/Philadelphie: John Benjamins Publishing Company, 114-132.

Lefeuvre, F. et Rossi-Gensane, N. (2015). Interrogation. Dans: P. Larrivée et F. Lefeuvre (éds), Projet Fracov. http://www.univ-paris3.fr/index-des-fiches-227311.kjsp?RH=1373703153287

Marchello-Nizia, C. (2012). L'oral représenté en français médiéval: un accès construit à une face cachée des langues mortes. Dans: C. Guillot, B. Combettes, A. Lavrentiev et É. Oppermann-Marsaux (éds), Le changement en français. Études de linguistique diachronique. Berne: Peter Lang, 247-264.

Mauger, G. (1968). Grammaire pratique du français d'aujourd'hui : langue parlée, langue écrite. Paris: Hachette.

Obenauer, H.-G. (1994). Aspects de la syntaxe A-barre. Effets d'intervention et mouvements des quantifieurs. Thèse de doctorat d'État, Université Paris VIII.

Price, G. (1971). The French Language: Past and Present. Londres: Arnold.

Quillard, V. (2000). Interroger en français parlé: études syntaxique, pragmatique et sociolinguistique. Thèse de doctorat, Université François Rabelais, Tours.

Riegel, M., Pellat, J.-C. et Rioul, R. (2009) [1994]. Grammaire méthodique du français. Paris: Presses Universitaires de France.

Rouayrenc, C. (2010). Le français oral. 2 - L'organisation et la réalisation de l'énoncé oral. Paris: Belin.

Sauvageot, A. (1964). Portrait du vocabulaire français. Paris: Larousse.

Söll, L. (1982). L'interrogation directe dans un corpus de langage enfantin. Dans: F.-J. Hausmann (éd.), Études de grammaire française descriptive. Heidelberg: Groos, 45-54.

Terry, R. M. (1970). Contemporary French Interrogative Structures. Montréal et Sherbrooke: Éditions Cosmos.

Trudgill, P. (1972). Sex, covert prestige and linguistic change in the urban British English of Norwich. Language in Society, 1: 179-195.

Tuaillon, G. (1975). Analyse syntaxique d'une carte linguistique: ALF 25: " Où vas-tu ? ». Revue de Linguistique Romane, 39: 79-96.

Cite this article: Rossi-Gensane N, Acosta Córdoba LF, Ursi B, and Lambert M (2021). Les structures interrogatives directes partielles fondées sur où dans les dialogues de romans français $d u X X^{e}$ siècle. Journal of French Language Studies 31, 169-191 https://doi.org/10.1017/S0959269520000253 